\title{
Effect of Nigella sativa seeds extracts on clinically important bacterial and fungal species
}

\begin{abstract}
Nigella sativa is a medicinal plant proven to have curing potential in many diseases. The present study was aimed to investigate the antibacterial and antifungal activities of $N$. sativa seeds extracts prepared in methanol, ethanol and water. The results of plant extracts were found to be promising as compared to culture sensitivity results of the antibiotics. Methanol extracts exhibited maximum activity against Staphylococcus aureus with $20 \mathrm{~mm}$ zone of inhibition. Ethanol extracts exhibited optimum activity against Proteus vulgaricus and Pseudomonas aeroginosa with $21 \mathrm{~mm}$ zone of inhibition. Water extract showed highest activity against Staphlococus aureus (16 $\mathrm{mm}$ zone of inhibition) and lowest activity against $P$. vulgaricus with $4 \mathrm{~mm}$ zone of inhibition. Against fungal isolates methanol extracts exhibited maximum activity against Penicilium digitatum $(20 \mathrm{~mm} \mathrm{ZI})$, whereas the ethanol extracts showed optimum activity against $P$. digitatum. Water extract showed highest activity against Rhizopus stolonifer with ZI recorded is $12 \mathrm{~mm}$ and showed least activity against Penicilium digitatum with ZI observed is $6 \mathrm{~mm}$. It was concluded that $N$. sativa seeds have significant antibacterial activity against gallbladder and infantile septicemia pathogens.
\end{abstract}

Keywords: nigella sativa, gallbladder, infantile septicemia, antibacterial, antifungal activity
Volume 5 Issue 4 - 2018

\author{
Syed Bilal Shah,' Syed Izhar Ali Shah, ${ }^{2}$ Lubna \\ Sartaj, ${ }^{3}$ Shah Zaman, ${ }^{4}$ Muhammad Bilal, \\ Muhammad Salman ${ }^{2}$ \\ 'State Key Laboratory of Microbial Metabolism, School of Life \\ Sciences and Biotechnology, Shanghai Jiao Tong University, China \\ ${ }^{2}$ Department of Microbiology, Abasyn University Peshawar, \\ Pakistan \\ ${ }^{3}$ Deprtment of Biochemistry, Abdul Wali Khan University \\ Mardan, Pakistan \\ ${ }^{4}$ School of Agriculture and Biology, Shanghai Jiao Tong University, \\ China
}

\section{Correspondence: Syed Bilal Shah, State Key Laboratory of Microbial Metabolism, School of Life Sciences and Biotechnology, Shanghai Jiao Tong University, Shanghai, 200240, \\ Tel +861312217629I, China, Email bilalshah@sjtu.edu.cn}

Received: August 01, 2018 | Published: August 16,2018

\section{Introduction}

Salmonella typhi and Salmonella paratyphi A are the main causative agents of enteric fever. ${ }^{1,2}$ The infection is common in those areas where unhygienic conditions prevailed. According to estimation round about 27 million people become victim of typhoid annually, of this 27 million 200000 people die. ${ }^{3}$ More than $95 \%$ of patients can be recovering by providing appropriate therapy. But study suggests that $2-5 \%$ of typhoid patients got chronic gallbladder infection. Gallbladder infection may be due to bile or directly from blood. Salmonella specie is acquiring resistance to antibiotics such as ciprofloxacin, ampicillin, cotrimoxazole, chloramphenicol and ceftriaxone, which has made difficult the management and treatment of enteric fever.

According to estimation, 4 million infants die every year around the world, out of which 1 million die because of infantile sepsis., Major causative agents of the infantile sepsis are Pseudomonas, E. coli, Proteus, Staphylococcus aureus and Klebsilla, out of these Pseudomonas and E. coli have developed multi drug resistance against cefotaxime, ceftrioxone, ceftzidine, gentamycin, amoxicillin and ampicillin. ${ }^{5}$ Mortality rate of neonatal septicemia is increasing due to emergence of antimicrobial drug resistance which is considered to be a challenge for researchers. ${ }^{5,6}$

Nigella sativa is a medicinal plant believed to have antitumor, antidiabetic, diuretic, gastroprotective, CNS depressant, antispasmolytic, anti-inflammatory, antioxidant, antimicrobial, anticonvulsant, antiurolithatic, antinociceptive, anxiolytic, hepatoprotective, nephroprotective, antihelminthic and immunomodulatory activitie. ${ }^{7} \mathrm{~N}$. sativa seeds extracts is used to treat coughs and remove renal stone and inhibit the growth of cancerous cells. It is used for the treatment of abdominal pain, polio and diarrhea. It also has anti-inflammatory and antioxidant activities. ${ }^{8}$ The oil of Nigella sativa have antihelminthic, antinematodal, Antischistosomal, antimicrobial and antiviral activities. ${ }^{9,10}$

\section{Materials and methods}

\section{Collection and processing of the seeds}

The seeds of $N$. Sativa were purchased from the local herbal market in Khyber bazaar Peshawar. The collected seeds were identified from the department of Botany, University of Peshawar. The healthy seeds were washed three times with distilled water and dried at $40^{\circ} \mathrm{C}$ overnight. After drying, the seeds were grounded into powder by an electric grinder and preserved. ${ }^{11}$

\section{Preparation of extracts}

A $100 \mathrm{~g}$ of powdered seeds material was dissolved in 1 liter of methanol, ethanol and distilled water and kept for $24 \mathrm{~h}$ at $25^{\circ} \mathrm{C}$. After $24 \mathrm{~h}$, the material was filtered and the filtrate was placed on water bath at $60^{\circ} \mathrm{C}$ for the evaporation of extra solvents. The extracts obtained were refrigerated at $4^{\circ} \mathrm{C}$ for further studies.

\section{Test bacterial and fungal cultures}

Various bacterial species namely Salmonella typhi, Salmonella paratyphi A, Escherichia coli, Staphylococcus aureus, Klebsilla pneumonia, Pseudomonas aeruginosa and Proteus vulgaricus, Staphlococcus epidermidis, Klebsiella oxytoca and Proteus mirabilis were isolated from the infantile sepsis and gallbladder patients in LRH, Peshawar. Fungal species including Aspergillus niger, Aspergillus fumigatus, Rhizopus stolonifer, Penicillium digitatum and acremonium alternatum were provided by the laboratories of LRH, Peshawar and Microbiology laboratory of Abasyn University Peshawar.

\section{Bacterial strains and antibiotics susceptibility test}

The effects of some commonly prescribed antibiotics were investigated against the test bacterial strains. For antibacterial assay, disc diffusion method was used on MHA. Standard antibiotics like 
Amoxycillin $(20 \mu \mathrm{g})$, Gentamicin $(10 \mu \mathrm{g})$, Clavulanic acid $(10 \mu \mathrm{g})$, Amikacin $(30 \mu \mathrm{g})$, Ceftrixone $(30 \mu \mathrm{g})$, Ciprofloxacin $(5 \mu \mathrm{g})$ and Vancomycin $(30 \mu \mathrm{g})$ discs were investigated for their antibacterial activities against all the tested bacteria. The antibiotics discs were placed on the inoculated agar plates and the plates were incubated at $37^{\circ} \mathrm{C}$ for $24 \mathrm{~h}$ followed by the measurement of inhibition zone.

\section{Antibacterial activity}

Agar well diffusion method was carried out to evaluate the antibacterial activity. The nutrient agar plates were prepared and test bacterial strains were inoculated on it. Then by means of $6 \mathrm{~mm}$ borer wells were produced on agar plates. From each extract, $100 \mu 1$ was transferred through micropipette into the wells in the inoculated agar plates. ${ }^{12}$ For better diffusion of the extracts before incubation; the plates were placed at $4^{\circ} \mathrm{C}$ for $3-4 \mathrm{~h} .{ }^{11}$ The bacterial inoculated plates were incubated at $37^{\circ} \mathrm{C}$ for $24 \mathrm{~h}$. After the completion of incubation period, zone of inhibition ( $\mathrm{mm}$ ) was measured. Each concentration of the extract was assessed for antibacterial activity in triplicate and their mean zone of inhibition was taken. ${ }^{13}$ A $50 \mu 1$ DMSO was used as negative control.

\section{Antifungal activity}

The potato dextrose agar (PDA) plates were prepared and the fungal strains like $A$. fumigatus, $A$. niger, $R$. stolonifer, $P$. digitatum and $A$. alternatum were spread on the PDA. A sterile $6 \mathrm{~mm}$ diameter cork borer was used to made holes in the inoculated plates. ${ }^{14} 50 \mu \mathrm{l}$ from each extract was applied through micropipette into the wells in the inoculated PDA plates. ${ }^{12}$ For better absorption of the extracts before incubation the plates were placed at 4centigrade for 3-4hours. ${ }^{11}$ The fungal inoculated plates were incubated at $25^{\circ} \mathrm{C}$ for $48 \mathrm{~h}$. After the completion of incubation period zone of inhibition was measured in $\mathrm{mm}$ by means of scale. Each concentration of the extracts was assessed for antifungal activity in triplicate and their mean zone of inhibition was taken. ${ }^{13}$

\section{Statistical data analysis}

Statistical analysis was performed using Microsoft Excel. All experiments were carried out in three different sets with each set in triplicates. The data are expressed as mean \pm SEM (standard error of the mean).

\section{Results}

\section{Culture sensitivity profile of bacterial and fungal species}

In this study, different antibiotics were used to assess the susceptibility pattern of the bacterial strains (Table 1). Results showed that the Ciprofloxacin antibiotic exhibited the highest zone of inhibition (28mm) against $E$. coli (Infantile sepsis isolate), whereas a maximum zone of inhibition was recorded against Salmonella typhi (Gallbladder isolate) by Vancomycin. In case of fungal strains, Acremonium alternatum was found to be the most sensitive by Nystatin followed by Rhizopus stolonifera and Penicilium digitatum.

Table I Culture sensitivity results of bacterial and fungal species by different antibiotics

\begin{tabular}{|c|c|c|c|c|c|c|c|}
\hline Microorganism & $\mathbf{C N}$ & CRO & CA & CIP & AMC & AK & $\mathbf{V N}$ \\
\hline & \multicolumn{7}{|c|}{ Diameter of inhibition zone (mm) } \\
\hline \multicolumn{8}{|c|}{ Infantile sepsis isolates (bacteria) } \\
\hline Escherichia coli & $12 \pm 0.22$ & $20 \pm 0.18$ & $9 \pm 0.07$ & $28 \pm 0.24$ & $5 \pm 0.02$ & $30 \pm 0.29$ & $32 \pm 0.36$ \\
\hline Staphylococcus aureus & $21 \pm 0.25$ & $9 \pm 0.10$ & $12 \pm 0.14$ & $13 \pm 0.10$ & $2 \pm 0.01$ & $8 \pm 0.09$ & $13 \pm 0.14$ \\
\hline Staphylococcus epidermidis & $19 \pm 0.34$ & $6 \pm 0.02$ & $10 \pm 0.13$ & $5 \pm 0.04$ & $3 \pm 0.02$ & $7 \pm 0.06$ & $11 \pm 0.08$ \\
\hline Klebsiella pneumonia & $11 \pm 0.12$ & $10 \pm 0.07$ & $8 \pm 0.17$ & $8 \pm 0.07$ & $4 \pm 0.05$ & $12 \pm 0.12$ & $14 \pm 0.15$ \\
\hline Klebsiella oxytoca & $9 \pm 0.09$ & $10 \pm 0.11$ & $7 \pm 0.05$ & $8 \pm 0.12$ & $6 \pm 0.08$ & $4 \pm 0.03$ & $16 \pm 0.24$ \\
\hline Proteus vulgaricus & $19 \pm 0.14$ & $14 \pm 0.12$ & $13 \pm 0.12$ & $20 \pm 0.35$ & $3 \pm 0.01$ & $17 \pm 0.18$ & $26 \pm 0.21$ \\
\hline Proteus mirabilis & $17 \pm 0.28$ & $14 \pm 0.04$ & $15 \pm 0.22$ & $19 \pm 0.42$ & $4 \pm 0.02$ & $16 \pm 0.14$ & $25 \pm 0.32$ \\
\hline Pseudomonas aeroginosa & $23 \pm 0.13$ & $10 \pm 0.16$ & $12 \pm 0.26$ & $21 \pm 0.28$ & $2 \pm 0.02$ & $8 \pm 0.08$ & $10 \pm 0.07$ \\
\hline \multicolumn{8}{|c|}{ Gallbladder isolates (bacteria) } \\
\hline Salmonella typhi & $13 \pm 0.26$ & $8 \pm 0.03$ & $10 \pm 0.32$ & $5 \pm 0.02$ & $7 \pm 0.01$ & $11 \pm 0.09$ & $19 \pm 0.19$ \\
\hline Salmonella paratyphi A & $10 \pm 0.41$ & $7 \pm 0.02$ & $12 \pm 0.06$ & ND & $9 \pm 0.12$ & $8 \pm 0.10$ & $17 \pm 0.51$ \\
\hline Fungal strains & FZ & $\mathbf{K Z}$ & NT & & & & \\
\hline Aspergillus niger & $2 \pm 0.22$ & $13 \pm 0.27$ & $16 \pm 0.11$ & & & & \\
\hline Aspergillus fumigatus & $11 \pm 0.22$ & $14 \pm 0.13$ & $12 \pm 0.09$ & & & & \\
\hline Rhizopus stolonifer & $3 \pm 0.22$ & $17 \pm 0.25$ & $18 \pm 0.18$ & & & & \\
\hline Penicilium digitatum & $2 \pm 0.22$ & $\mathrm{ND} \pm 0.12$ & $17 \pm 0.15$ & & & & \\
\hline Acremonium alternatum & $1 \pm 0.05$ & $15 \pm 0.21$ & $19 \pm 0.23$ & & & & \\
\hline
\end{tabular}

CN, Gentamicin; CRO, Ceftriaxone; CA, Clavulanic acid; CIP, Ciprofloxacin;AMC,Amoxicillin;AK, Amikacin; VN, Vancomycin; FZ, Fluconazole; KZ, Ketoconazole; NT, Nystatin 


\section{Antibacterial and antifungal activity of plant extracts}

Antibacterial activity of the $N$. sativa seeds extracts was determined against the selected bacterial species and ZI were measured as shown in Table 2. The ZI recorded for E. coli was 19, 18 and $12 \mathrm{~mm}$ for ethanol, methanol and water extract, respectively, which showed that $E$. coli is sensitive to the seeds extracts. The ZI measured for $S$. aureus was 19,20 and $16 \mathrm{~mm}$ for ethanol, methanol and water extracts, respectively, evidencing the potency of the extracts against $S$. aureus. Contrary to $S$. aureus, $S$. epidermidis showed resistance towards ethanol extract and the ZI observed was 3, 11 and $15 \mathrm{~mm}$ for ethanol, methanol and water extracts, respectively. K. pneumonia also showed resistance towards ethanol extract and $K$. oxytoca and was found sensitive towards ethanol $(12 \mathrm{~mm})$ and water $(14 \mathrm{~mm})$ but was found resistant towards methanol with no detected ZI. P. vulgaricus was sensitive towards ethanol and methanol extracts but resistant towards water extract. P. mirabilis and P. aeuroginosa were found sensitive towards all the extracts with different ZI. Similarly, the investigated plant extracts also exhibited considerable antimicrobial effects against all the tested fugal strains and therefore different ZI.

Table 2 Antimicrobial and antifungal potentiality of $\mathrm{N}$. sativa seeds extracts against different strains

\begin{tabular}{|c|c|c|c|c|}
\hline Microorganism & Ethanol & Methanol & Water & DMSO \\
\hline & \multicolumn{4}{|c|}{ Diameter of inhibition zone (mm) } \\
\hline \multicolumn{5}{|c|}{ Infantile sepsis isolates (bacteria) } \\
\hline Escherichia coli & $19 \pm 0.13$ & $18 \pm 0.17$ & $12 \pm 0.12$ & ND \\
\hline Staphylococcus aureus & $19 \pm 0.26$ & $20 \pm 0.43$ & $16 \pm 0.27$ & ND \\
\hline Staphylococcus epidermidis & $3 \pm 0.03$ & $11 \pm 0.09$ & $15 \pm 0.17$ & ND \\
\hline Klebsiella pneumonia & $2 \pm 0.01$ & $16 \pm 0.21$ & $14 \pm 0.14$ & ND \\
\hline Klebsiella oxytoca & $12 \pm 0.21$ & ND & $14 \pm 0.22$ & ND \\
\hline Proteus vulgaricus & $21 \pm 0.43$ & $18 \pm 0.26$ & $4 \pm 0.33$ & ND \\
\hline Proteus mirabilis & $18 \pm 0.16$ & $14 \pm 0.11$ & $13 \pm 0.63$ & ND \\
\hline Pseudomonas aeruginosa & $21 \pm 0.18$ & $19 \pm 0.23$ & $11 \pm 0.15$ & ND \\
\hline \multicolumn{5}{|c|}{ Gallbladder isolates (bacteria) } \\
\hline Salmonella typhi & $14 \pm 0.19$ & $11 \pm 0.08$ & $10 \pm 0.37$ & ND \\
\hline Salmonella paratyphi A & $13 \pm 0.09$ & $12 \pm 0.16$ & $9 \pm 0.07$ & ND \\
\hline \multicolumn{5}{|l|}{ Fungal strains } \\
\hline Aspergillus niger & $15 \pm 0.23$ & $14 \pm 0.073$ & $9 \pm 0.01$ & ND \\
\hline Aspergillus fumigatus & $17 \pm 0.27$ & $19 \pm 0.36$ & $7 \pm 0.03$ & ND \\
\hline Rhizopus stolonifera & $16 \pm 0.11$ & $15 \pm 0.05$ & $12 \pm 0.01$ & ND \\
\hline Penicilium digitatum & $18 \pm 0.34$ & $20 \pm 0.29$ & $6 \pm 0.01$ & ND \\
\hline Acremonium alternatum & $13 \pm 0.22$ & $17 \pm 0.13$ & $11 \pm 0.06$ & ND \\
\hline
\end{tabular}

\section{Discussion}

Due to irrational use of antibiotics, prolonged hospitalization and improper curing and preventive procedures to control the pathogenic microorganisms, infectious agents are acquiring resistance to commercial antibiotics. ${ }^{15,16}$ Moreover the antibiotics are not so much effective and if effective they are either unavailable or expensive and also have certain level of toxic effects on human body. ${ }^{17,18}$ Therefore, researchers are trying to discover such compounds in plants which are effective, less expensive and have no or less toxicity. In order to overcome the resistance problem and discover drugs which are effective, less expensive, less toxic and easily available we focused on medicinal plants and designed the current study by using $N$. sativa seeds extracts against Enteric fever is caused by $S$. typhi and $S$. paratyphi A. In most of the cases, Salmonella infection can be treated by proper therapy but occasionally Salmonella enter into gall bladder and cause serious gallbladder complications which are difficult to cure. Death rate from infantile sepsis is increasing because of the resistance problem acquired by pathogens towards available drugs like cefotaxime, ceftrioxone, ceftzidine, gentamycin, amoxicillin and ampicillin which is challenging task for the researchers. Therefore, our study aimed to evaluate the antibacterial and antifungal activities of a medicinal plant known as $N$. sativa against gallbladder, infantile sepsis patient's isolates and some clinically important pathogenic fungi.

Eight bacterial species were isolated from infantile septicemia patients and two species were isolated from gall bladder patients and then culture sensitivity profile was determined for these isolated strains. K. pnemonia and K. oxytoca showed resistance towards $86 \%$ of the antibiotics used. Similarly P. auroginosa exhibited $71 \%, S$. epidermidis $71 \%$, S. typhi $28 \%$, S. paratyphi $28 \%$, P. vulgaricus $14 \%$, P. miribilis $14 \%$, E. coli $42 \%$ and $S$. aureus $57 \%$ resistance against antibiotics applied in the study. Our results for culture sensitivity tests showed little variations with research performed by ${ }^{19}$ showing that $P$. aeroginosa and $S$. aureus were $50 \%$ resistant, S. typhi $71.4 \%$, k. pnemonia $85.7 \%$ and E. coli and P. vulgaricus $78.6 \%$ resistant to various antibiotics. ${ }^{20}$ Proved that Klebsilla has $100 \%, 76.3 \%, 68.4 \%$ 
and $28.7 \%$ resistant against Amoxicillin, gentamicin, ciprofloxacin and ceftriaxone, respectively. The results also indicate that $E$. coli showed 96\%, 83.4\%, 76.4\% and 32.5\% against Amoxicillin, gentamicin, ciprofloxacin and ceftriaxone, respectively. Similarly, resistance exhibited by Proteus specie against antibiotics used were Amoxicillin (95\%), gentamicin (77.2\%), ciprofloxacin (65\%) and ceftriaxone $(23.4 \%)$.

Ethanolic, methanolic and water extracts of the seeds were evaluated for their antibacterial activity. All the extracts showed significant results against the tested strains. Ethanol extract was proven to have more effective as compared to methanol and water extracts. Ethanol extract exhibited maximum activity against $P$. auroginosa with $21 \mathrm{~mm} \mathrm{ZI} \mathrm{and} \mathrm{minimum} \mathrm{activity} \mathrm{against} K$. pnemonia with $2 \mathrm{~mm}$ ZI. It was concluded that aqueous extracts have less activity as compared to ethanol and methanol extracts and the reason might be that water can only extract polar compounds while ethanol and methanol are amphiphilic in nature and can extracts organic and inorganic compounds both. Similarly all the three extracts showed promising results against other tested isolates and their activity was impressing as compared to the activity of commercial antibiotics. Our study concluded that $N$. sativa seed extracts prepared in ethanol, methanol and distilled water has antimicrobial activity against both bacterial and fungal strains isolated from gallbladder and infantile sepsis patients.

\section{Competing interests}

The author declares that there is no conflict of interests.

\section{Acknowledgements}

None.

\section{Author's contributions}

SIAS performed the experimentation; SBS wrote the first draft and helped in giving critical view of manuscript writing. LS and MB designed the study, wrote the manuscript and carried out the statistical analysis. MS participated in the collection of different plant parts and helped in experimentation. SBS also participated in taxonomist assessment for plant identification and in writing manuscript. All authors read and approved the final manuscript.

\section{Funding}

The author(s) received no financial support for the research, authorship, and/or publication of this article. However the practical work was performed at Department of Microbiology and Biotechnology, Abasyn University, Peshawar Pakistan.

\section{References}

1. Parry CM, Hien TT, Dougan G, et al. Typhoid fever. $N$ Engl J Med. 2002;347(22):1770-1782.

2. Powell WA, Catranis CM, Maynard CA. Design of self-processing antimicrobial peptides for plant protection. Lett Appl Microbiol. 2000;31(2):163-168.

3. Adabara B, Ezugwu U, Momojimoh A, et al. The prevalence and antibiotics susceptibility pattern of salmonella typhi among patients attending a Military Hospital in Minna. Adv Prev Med. 2012;5:88-92.

4. Ahmed SK, Mohammed IG, El Bari EO, et al. In vitro and microbiological activity of ethanolic seeds extracts of Nigella sativa (Linn) in Sudan. Afr. J. Microbiol. Res. 2015;9:788-792.

5. Rahman S, Hameed A, Roghani MT, et al. Multidrug resistant neonatal sepsis in Peshawar. Arch Dis Child Fetal Neonatal Ed. 2002;87(1):F5254 .

6. Shah SB, Parveen Z, Bilal M, et al. Assessment of antimicrobial, antioxid ant and cytotoxicity properties of Camellia sinensis L. Pak $J$ Pharm Sci. 2018;31(4):1285-1291.

7. Padmaa, MP. Nigella sativa Linn.-A comprehensive review. Indian J Nat Prod Resour. 2010;1(4):409-429.

8. Enomoto S, Asano R, Iwahori Y, et al. Hematological studies on black cumin oil from the seeds of Nigella sativa L. Biol Pharm Bull. 2001;24(3):307-310.

9. Thaver D, Ali SA, Zaidi AK. Antimicrobial resistance among neonatal pathogens indeveloping countries. Pediatr Infect Dis J. 2009;28(1 Suppl):S19-21.

10. Saleem ML. Immunomodulatory and therapeutic properties of the Nigella sativa L. seed. Int Immunopharmacol. 2005;5(13-14):17491770 .

11. Alam MM, Yasmin M, Nessa J, et al. Antibacterial activity of chloroform and ethanol extracts of cumin seeds (Nigella sativa) against multi-drug resistant human pathogens under laboratory conditions. $J$. Med. Plants Res. 2010; 18:1901-1905.

12. Ibtisam MA. Antimicrobial activity of ethanolic extracts from some medicinal plants. Aust. J. basic. 2011;5:678-683.

13. Manjulika Y, Sanjukta C, Deepak KY, et al. Improved antibacterial efficacy of a novel formulation- synergistic effect of herbs. Int. J. Pharm. Biol. Sci. 2014;4:36-39.

14. Arunkumar, S.; Muthuselvam, M. Analysis of phytochemical constituents and antimicrobial activities of Aloe vera L. against clinical pathogens. World journal of agricultural sciences. 2009;5(5):572-576.

15. Akhtar MS, Riffat S. Field trial of Saussurea lappa roots against nematodes and Nigella sativa seeds against cestodes in children. J Pak Med Assoc. 1991;41(8):185-187.

16. Anjali Soni, Sheetal Sosa. Phytochemical analysis and free radical scavenging potential of herbal and medicinal plant extracts. $J$ Pharmacogn Phytochem. 2013;2(4):22-29.

17. Deepa P, Kaleena PK, Valivittan K, et al. Phytochemical screening and antimicrobial activity of Sansevieria roxburghiana Schult and Schult. F. Middle-East J. Sci. 2011;10(4):512-518.

18. Gracelin DHS, Britto ADJ, Kumar PBJR. Antibacterial screening of a few medicinal ferns against antibiotic resistant phytopathogen. Int $J$ Pharm Sci Res. 2012;3:868-873.

19. Ishaq MS, Hussain MM, Afridi MS. et al. In vitro phytochemical, antibacterial, and antifungal activities of leaf, stem, and root extracts of Adiantum capillus veneris. Sci World J. 2014;1-7.

20. Naveed M, Khan TA, Ali I, et al. In vitro antibacterial activity of Cannabis sativa leaf extracts to some selective pathogenicbacterial strains. Int J Biosci. 2014;4(4):65-70. 